\title{
Analgesia with abdominal wound catheters post laparotomy in adults
}

J Rudiger, T Barnes, A Kapuscinska, S O'Manth, S Goodge, A Belgaumkar, E Syrrakou - East Surrey Hospital, Redhill, UK - ESRA - Sept. 2019

\section{Introduction}

Epidural analgesia has long been considered the 'gold standard for pain relief following major abdominal surgery. Epidural analgesia is not without risk, however (1). In some patients epidural analgesia may even be contraindicated.

Continuous infusion of local anaesthetic via abdominal wound catheters have been introduced to our Trust as an alternative to thoracic epidurals. (Insertion technique: see diagram below on the right). There is evidence that wound catheters may be comparable in terms of post-op pain scores to epidural analgesia (2). Wound catheters have also been shown to decrease opioid consumption and side effects including nausea, vomiting and pruritis compared with epidural analgesia (3).

Wound catheter infusions can be used as an alternative to thoracic epidurals for pain relief after emergency or elective surgery:
1. Midline laparotomy
2. Large umbilical hernia repair
3. Large incisional hernia repair
4. Open cholecystectomy

Contraindications: patient refusal, allergy to local anaesthetics, confusion, signs of an infection at the catheter insertion side

\section{Objective}

To compare post-operative pain scores and opioid consumption in patients with and without abdominal wall wound catheters following major abdominal surgery. (This is not a comparison of wound catheters vs. epidural analgesia)

\section{Method}

A prospective audit was undertaken between 03-12 / 2018 of randomly selected patients with wound catheters (19) and from a control group of patients who received neither wound catheters nor epidural (9) following laparotomies. All had Morphine-PCAs.

Data collected: 1. VAS pain scores (out of 10), 2. opioids given (until day 5), 3. Intra-operative analgesia (spinal, opioids), 4. details of local anaesthetic given via wound catheter peri-operatively.

\section{Results}

In the wound catheter group the following results were found:

- Lower median post-op VAS scores at rest (d1: 2 vs.4) and on movement (d1: 5 vs. 8) up to 3 days post-op (Graph on the left)

- Lower median PCA morphine requirement (66mg vs 79mg)

Only $29 \%$ of patients received a spinal injection for visceral pain.

Initial Levobupivacaine-boluses via wound catheters: $60 \mathrm{ml}(32 \%), 40 \mathrm{ml}(26 \%), 20 \mathrm{ml}(5 \%)$, not documented (37\%)

Post-operative pain scores

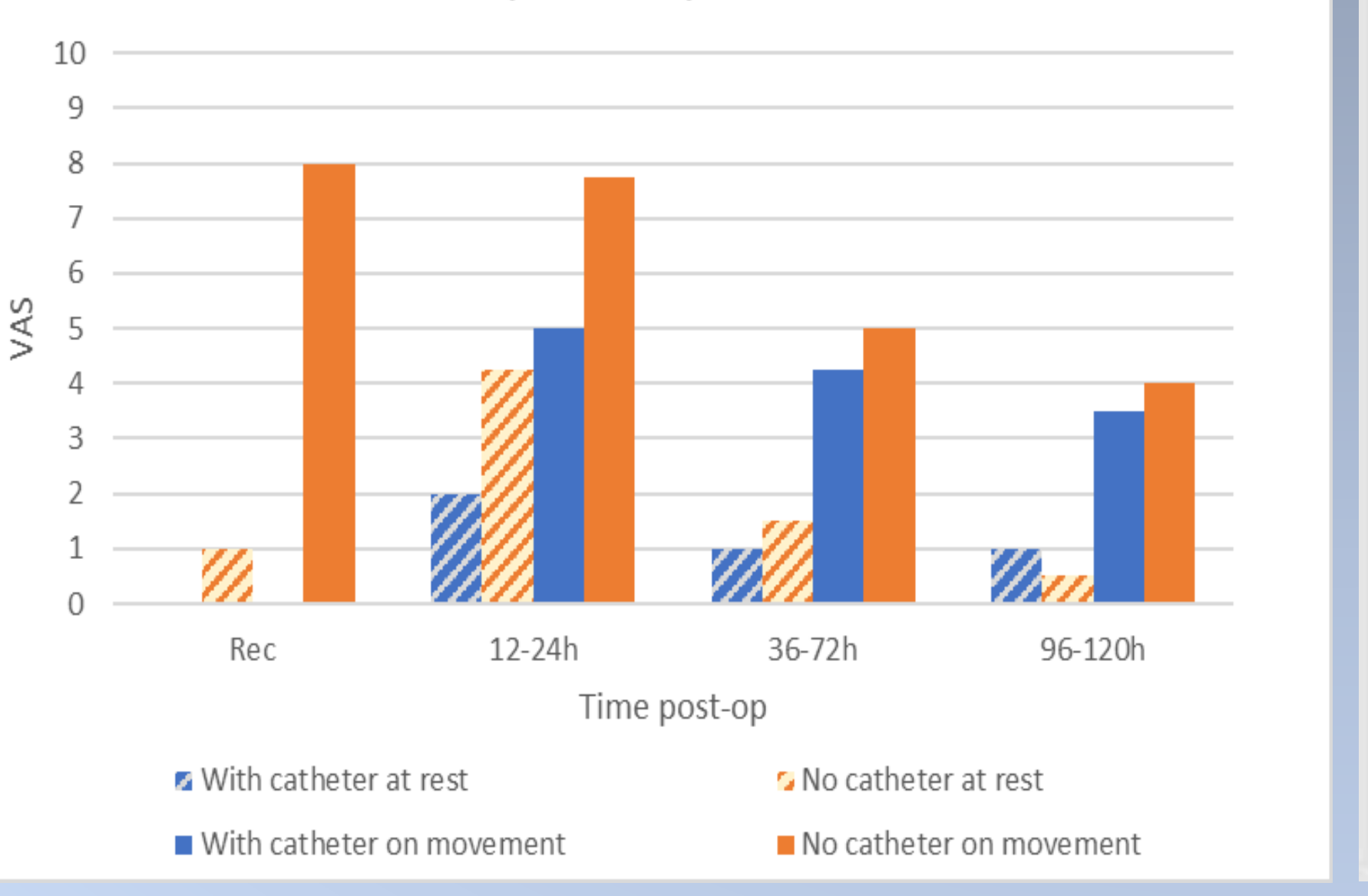

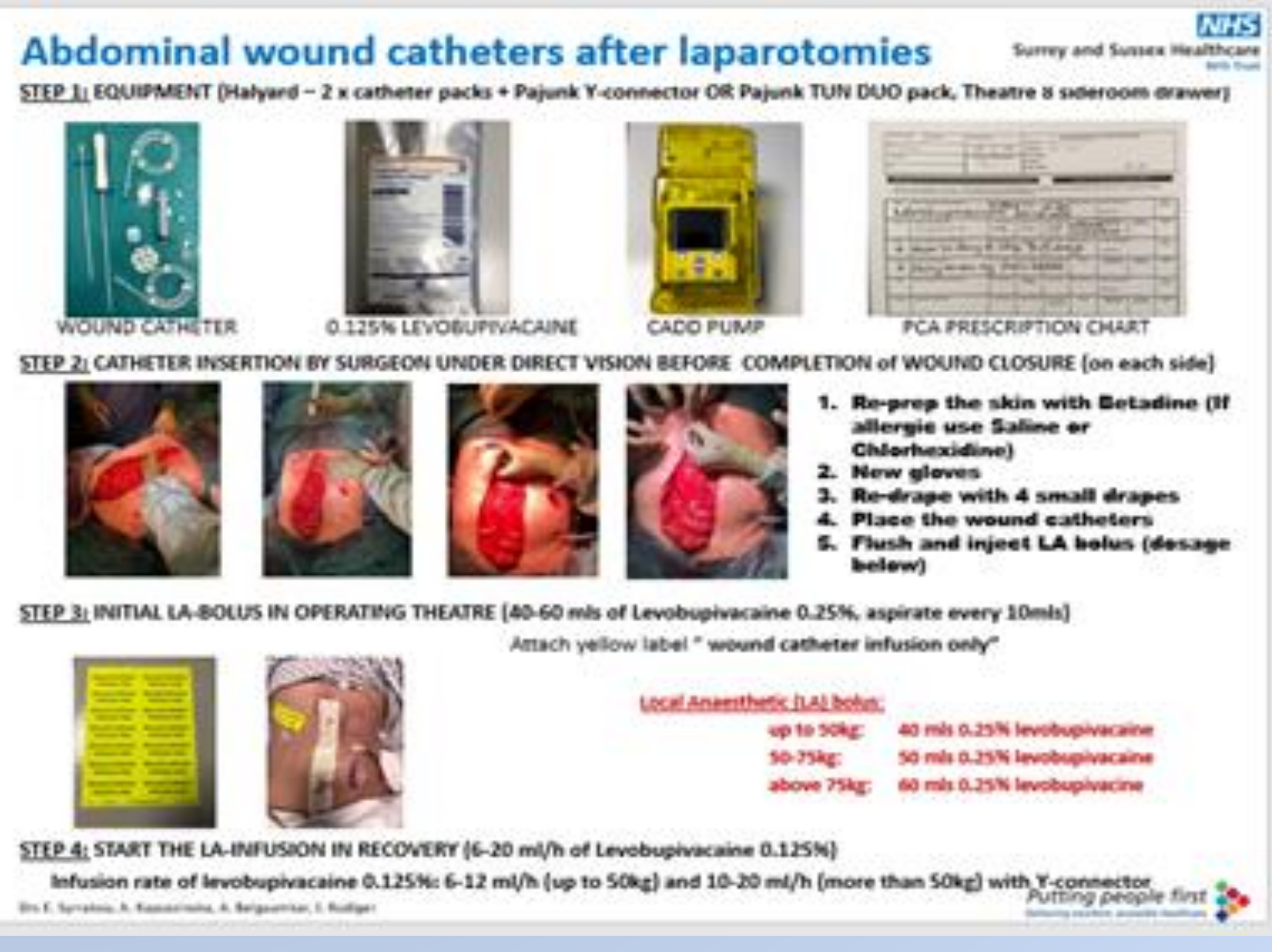

Conclusion: Post-operative pain scores and opioid consumption were reduced in patients with abdominal wound catheters. 